\title{
NONTRIVIAL COMPACT BLOW-UP SETS OF SMALLER DIMENSION
}

\author{
MAYTE PÉREZ-LLANOS AND JULIO D. ROSSI
}

\begin{abstract}
We provide examples of solutions to parabolic problems with nontrivial blow-up sets of dimension strictly smaller than the space dimension. To this end we just consider different diffusion operators in different variables, for example, $u_{t}=\left(u^{m}\right)_{x x}+u_{y y}+u^{m}$, or, $u_{t}=\left(\left|u_{x}\right|^{p-2} u_{x}\right)_{x}+u_{y y}+u^{p-1}$. For both equations, we prove that there exists a solution that blows up in the segment $B(u)=[-L, L] \times\{0\} \subset \mathbb{R}^{2}$.
\end{abstract}

\section{INTRODUCTION.}

Our main concern in this note is to find examples of blowing up solutions to parabolic problems which exhibit a nontrivial compact blow-up set of dimension strictly less than the space dimension.

For many parabolic equations it is well known that solutions develop singularities in finite time no matter how smooth the initial data are. A special case of this occurs if the $L^{\infty}$-norm of the solution goes to infinity in finite time, that is, there exists a finite time $T$ such that $\lim _{t \rightarrow T}\|u(\cdot, t)\|_{\infty}=+\infty$. This phenomenon is called blow-up and has been object of active research in recent years, see the survey [5], the book [8] and references therein. An important issue arising from the study of blow-up problems is the spatial structure of the set where the solution becomes unbounded, that is, the blow-up set. More precisely, the blow-up set of a solution $u$ that blows up at time $T$ is defined as

$$
B(u)=\left\{x ; \text { there exist } x_{n} \rightarrow x, t_{n} \nearrow T \text {, with } u\left(x_{n}, t_{n}\right) \rightarrow \infty\right\} .
$$

A problem which has attracted some attention in the literature is the identification of possible blow-up sets. There are several cases where the blow-up set is known to be a single point (single point blow-up), for example for $u_{t}=\Delta u+u^{p}$ with $p>1,[1]$. Also, it can be a proper subset of the spatial domain of the same dimension (regional blow-up), for example for $u_{t}=\Delta u^{m}+u^{m}$, with $m>1$, see [2], [3], or the whole space (global blow-up), for example for $u_{t}=\Delta u^{m}+u^{p}$ with $(1<p<m)$, see [8]. Moreover, considering radial solutions for $u_{t}=\Delta u+u^{p}$ it is easy to construct an example with blow-up set a sphere, $B(u)=\{|x|=R\}$. In addition, from the results of [10] and [11], some regularity of the blow-up set is known for solutions of the above mentioned equation $u_{t}=\Delta u+u^{p}$. Hence, up to now, we have as possible blow-up sets: isolated points, the whole space, balls and spheres.

As we will show here, many other examples of blow-up sets are possible. For instance, we can find a parabolic equation with a solution with blow-up set a segment in $\mathbb{R}^{2}, B(u)=[-L, L] \times\{0\}$. In general, given any dimensions, $N, M$,

Key words and phrases. Blow-up sets, $p$-Laplacian, porous media.

2000 Mathematics Subject Classification. 35B40, 35K65, 35J25, 35J60. 
there exists a solution to a parabolic problem in $\mathbb{R}^{N+M}$, with blow-up set a disc, $B(u)=B(0, L) \times\{0\}$, where $B(0, L)$ is the $N$-dimensional ball in $\mathbb{R}^{N}$ of radius $L$. To construct these examples we propose to look at the following problems,

$$
u_{t}=\Delta_{x} u^{m}+\Delta_{y} u+u^{m}, \quad \text { in } \mathbb{R}^{N+M} \times(0, T),
$$

with $m>1$ and

$$
u_{t}=\nabla_{x}\left(\left|\nabla_{x} u\right|^{p-2} \nabla_{x} u\right)+\Delta_{y} u+u^{p-1}, \quad \text { in } \mathbb{R}^{N+M} \times(0, T),
$$

with $p>2$. Here the subindex denotes derivatives with respect to the first $N$ variables $(x)$ or with respect to the last $M$ variables $(y)$. Remark that the diffusion operator in (1.1) is a combination of the porous medium and the usual Laplacian operator in different space variables, while the operator in (1.2) is a combination of the $p$-Laplacian and the Laplacian.

Theorem 1.1. There exists $L>0$ such that, for any given two sets of points $\left\{y_{1}, \ldots, y_{k}\right\} \in \mathbb{R}^{M}$, and $\left\{x_{1}, \ldots, x_{j}\right\} \in \mathbb{R}^{N}$ with $\left|x_{i}-x_{j}\right|>2 L$ there exists a solution to (1.2) (or to (1.1)) whose blow-up set is

$$
B(u)=\bigcup_{i=1}^{j} B\left(x_{i}, L\right) \times\left\{y_{1}, \ldots, y_{k}\right\} .
$$

Note that this result provides examples of nontrivial blow-up sets of dimension smaller than the dimension of the ambient space.

\section{Proof of the Main Result.}

Proof of Theorem 1.1. The proof of the Theorem 1.1 is extremely simple, we just have to look for an adequate self-similar solution.

In the analysis of blow-up problems for parabolic equations that enjoy some scaling invariance, self-similar profiles are used to study the fine asymptotic behavior of a solution near its blow-up time. It often happens that the spatial shape of the solution near blow-up is close to a self-similar profile, see, for instance, [2], [3] and $[5]$.

Accordingly, when dealing with our problems (1.1) and (1.2) we will consider self-similar solutions of the special form

$$
u(x, y, t)=\varphi(x) \psi(y, t) .
$$

Let us begin considering (1.1). If $u$ is a solution of the form (2.1) we obtain that $\varphi$ and $\psi$ must solve the following elliptic and parabolic problems, respectively,

$$
\varphi(x)=\Delta_{x} \varphi^{m}(x)+\varphi^{m}(x), \quad x \in \mathbb{R}^{N},
$$

and

$$
\psi_{t}(y, t)=\Delta_{y} \psi(y, t)+\psi^{m}(y, t), \quad(y, t) \in \mathbb{R}^{M} \times(0, T) .
$$

Remark that the former equation is the heat equation with a source given by the term $\psi^{m}$, which has blowing up solutions if $m>1$ for large initial conditions.

Now, we just observe that the blow-up set of a solution $u(x, y, t)$ of the form (2.1) is given by

$$
B(u)=\operatorname{supp}(\varphi) \times B(\psi),
$$

where $B(\psi)$ is the blow-up set of $\psi$. The set $B(\psi)$ is known to be a finite set of points and generically a single point, see [1] and [6], [7], [9]. Hence, to find the 
desired set $B(u)$ we need to identify the support of $\varphi$. The determination of this support is based on the fact that there exists a unique radial solution to (2.2) with $\varphi^{m} \in H^{1}\left(\mathbb{R}^{N}\right)$, see [4]. This fact is simple to prove, since looking for radial solutions reduces (2.5) to an O.D.E. problem. In one space dimension, $N=1$, the solution is given by the explicit formula

$$
\varphi(x)=\left(\frac{2 m}{m^{2}-1} \cos ^{2}\left(\frac{m-1}{2 m} x\right)_{+}\right) .
$$

In general, it holds that a solution of (2.2) (in the natural energy space $H^{1}\left(\mathbb{R}^{N}\right)$ ) consists of a union of a finite number of separated copies of the radial profile centered at some points $x_{1}, \ldots, x_{j}$ with $\left|x_{i}-x_{j}\right|>2 L$, where $L$ is the radius of the support of the unique radial solution, see [4].

Therefore, we conclude from (2.4) that there exists a solution to (1.1) with blowup set given by

$$
B(u)=\bigcup_{i=1}^{j} B\left(x_{i}, L\right) \times\left\{y_{1}, \ldots, y_{k}\right\} .
$$

A similar analysis can be done for solutions to (1.2). In this case, if we consider as we previously did a solution of the form

$$
u(x, y, t)=\varphi(x) \psi(y, t)
$$

we get that $\varphi$ and $\psi$ solve

$$
\varphi(x)=\nabla_{x}\left(\left|\nabla_{x} \varphi\right|^{p-2} \nabla_{x} \varphi(x)\right)+\varphi^{p-1}(x), \quad x \in \mathbb{R}^{N}
$$

and

$$
\psi_{t}(y, t)=\Delta_{y} \psi(y, t)+\psi^{p-1}(y, t), \quad(y, t) \in \mathbb{R}^{M} \times(0, T) .
$$

The last part of the proof runs exactly as before noticing that compactly supported radial solutions to (2.5) are known to exist, see [8].

Acknowledgements MP is partially supported by DGICYT grant PB94-0153 (Spain) and JDR by ANPCyT PICT 5009, UBA X066 and CONICET (Argentina).

\section{REFERENCES}

[1] X. Y. Chen and H. Matano. Convergence, asymptotic periodicity and finite point blow up in one-dimensional semilinear heat equations. J. Differential Equations, Vol. 78, (1989), 160-190.

[2] C. Cortázar, M. Del Pino and M. Elgueta. On the blow-up set for $u_{t}=\Delta u^{m}+u^{m}, m>1$. Indiana Univ. Math. J. Vol. 47(2), (1998), 541-561.

[3] C. Cortázar, M. Del Pino and M. Elgueta. Uniqueness and stability of regional blow-up in a porous-medium equation. Ann. Inst. H. Poincaré Anal. Non Linéaire. Vol. 19(6), (2002), 927-960.

[4] C. Cortázar, M. Elgueta and P. Felmer. Symmetry in an elliptic problem and the blow-up set of a quasilinear heat equation. Comm. Partial Diff. Eq. Vol. 21(3\&4), (1996), 507-520.

[5] V. Galaktionov; J. L. Vázquez. The problem of blow-up in nonlinear parabolic equations, Discrete Contin. Dynam. Systems A 8 (2002), 399-433.

[6] F. Merle. Solution of a nonlinear heat equation with arbitrarily given blow-up points. Comm. Pure Appl. Math. Vol. XLV, (1992), 263-300.

[7] C. E. Muller and F. B. Weissler. Single point blow up for a general semilinear heat equation. Indiana Univ. math. J., Vol. 34, (1983), 881-913.

[8] A. Samarski, V. A. Galaktionov, S. P. Kurdyunov and A. P. Mikailov. Blow-up in quasilinear parabolic equations. Walter de Gruyter, Berlin, (1995). 
[9] F. B. Weissler. Single point blow up of semilinear initial boundary value problems. J. Differential Equations, Vol. 55, (1984), 204-224

[10] H. Zaag. Determination of the curvature of the blow-up set and refined singular behavior for a semilinear heat equation. Preprint.

[11] H. Zaag. On the regularity of the blow-up set for semilinear heat equations. Ann. Inst. H. Poincar Anal. Non Linaire. Vol. 19(5), (2002), 505-542.

Mayte Pérez-Llanos

Departamento de Matemáticas, U. Carlos iII de Madrid,

28911 Leganés, SPAIN.

E-mail address: mtperez@math.uc3m.es

Julio D. Rossi

Depto. Matemática, FCEyn UBA (1428)

Buenos Aires, Argentina.

E-mail address: jrossi@dm.uba.ar 